\title{
L'HOME L'Homme
}

Revue française d'anthropologie

185-186 | 2008

L'anthropologue et le contemporain : autour de Marc Augé

\section{"C'était une sorte de bulle..."}

Jean-Louis Boutillier

\section{OpenEdition}

\section{Journals}

Édition électronique

URL : http://journals.openedition.org/lhomme/24101

DOI : 10.4000//homme.24101

ISSN : 1953-8103

Éditeur

Éditions de l'EHESS

\section{Édition imprimée}

Date de publication : 1 janvier 2008

Pagination : 33-37

ISSN : 0439-4216

\section{Référence électronique}

Jean-Louis Boutillier, " "C'était une sorte de bulle..." », L'Homme [En ligne], 185-186 | 2008, mis en ligne le 01 janvier 2010, consulté le 02 mai 2019. URL : http://journals.openedition.org//homme/24101 ; DOI : 10.4000/lhomme.24101

\section{(c) École des hautes études en sciences sociales}




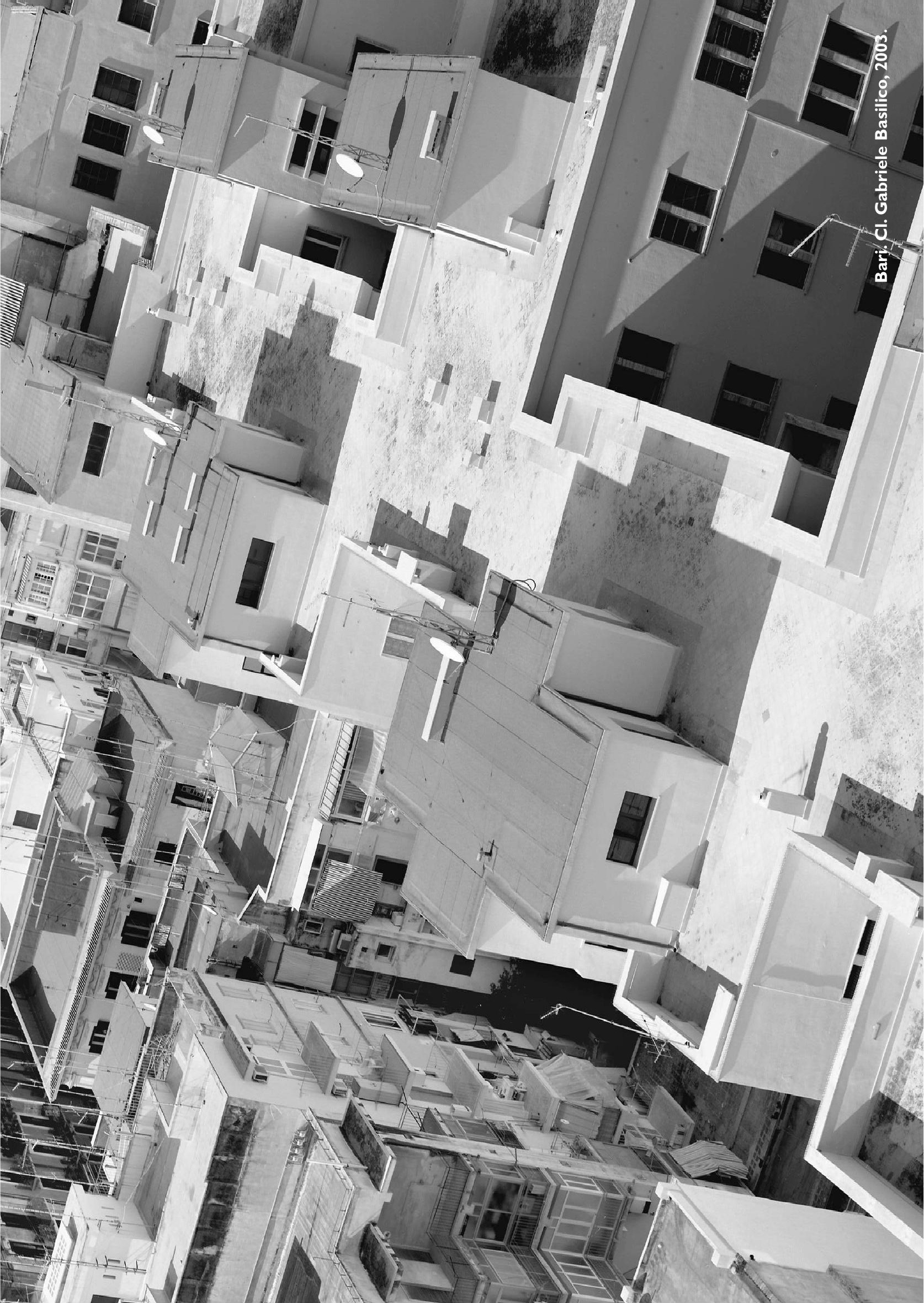




\title{
"C'était une sorte de bulle..."
}

\author{
Jean-Louis Boutillier
}

\begin{abstract}
C)
EST DANS LES SOUS-SOLS du Musée de l'Homme que j’ai débuté dans le métier. Après l'année de formation passée à suivre les cours de l'Institut d'ethnologie, on faisait un stage chez André Leroi-Gourhan - un stage de je ne sais plus quoi au juste: j’ai le souvenir qu'on dessinait des silex de façon mortelle, sur des planches...
\end{abstract}

Un jour, un homme très distingué, très allure vieil officier des années 1930, s'est présenté et a dit qu'il recherchait un jeune chercheur qui avait des notions d'économie et d'ethnologie parce qu'il était alors question de construire et de développer des usines pour traiter le palmier à huile en Côte-d'Ivoire, et qu'il était important de comprendre les rapports qui pouvaient sinstaurer entre l'édification de ce type d'usine et les populations locales. Le personnage distingué en question n'était autre que le gouverneur Hubert Deschamps qui avait à l'époque la haute main sur les sciences humaines de l'ORSTOM. Je me suis donc retrouvé recruté pour six mois, en 1954, par l'ORSTOM, et je suis parti en même temps que Marguerite Dupire qui, elle aussi, avait été recrutée pour les mêmes raisons. D'où "la palmeraie adioukrou", livre que nous avons écrit en commun ${ }^{1}$, tout en ne partageant pas les lits-picots que, au grand étonnement des gens des villages où nous séjournions, nous avions installés dans des cases différentes. Le problème était simple: on avait construit une huilerie énorme, avec des camions qui passaient chaque jour sur les pistes, mais on ne comprenait pas pourquoi les gens alentour ne vendaient pas les régimes de palmier à l'usine. En ce temps-là, c'était encore l'Union française.

1. Marguerite Dupire \& Jean-Louis Boutillier, Le Pays adioukrou et sa palmeraie (Basse Côted'Ivoire), Paris Éd. de l'ORSTOM, 1958 ("L'Homme d'outre-mer»). Propos recueillis en mai 2007, et annotations, par Jean-Pierre Dozon et Jean Jamin. 
Claude Tardits avait été recruté selon les mêmes modalités, mais lui travaillait sur la palmeraie du Dahomey (Bénin actuel).

Il faut dire qu'à cette époque, l'ethnologie offrait certains débouchés. C'est ainsi qu'à son séminaire à l'École pratique des hautes études (Ve section), Claude Lévi-Strauss, à la fin d'une séance, avait fait état de l'offre d'un poste d'ethnologue pour la mission chargée de l'aménagement de la moyenne vallée du Sénégal - offre à laquelle javais répondu, et poste auquel je fus également recruté après mes missions de l'ORSTOM en Côte-d'Ivoire ${ }^{2}$.

J'ai rencontré plus tard un expert de la FAO qui m'a employé pour entreprendre une des premières enquêtes "nutrition-niveau de vie en Côte-d'Ivoire". Et c'est à ce moment-là que jai commencé mon étude sur Bongouanou qui a duré dix-huit mois, et a montré le grand boom du cacao qui se profilait ${ }^{3}$. On se trouvait encore en pleine période coloniale, disons quil y avait encore tout l'appareil administratif colonial même si Houphouët-Boigny commençait à avoir de l'influence et avait remporté les élections de janvier 1956 avec le Rassemblement démocratique africain (RDA). Avant que je parte en Côted'Ivoire, Georges Balandier mavait donné le nom de Bernard Dadié, qui était bibliothécaire à l'IFAN [Institut français d'Afrique noire], et c'est dès cette époquelà que je suis devenu très ami avec lui.

En 1964, jai été affecté par le directeur général de l'ORSTOM à Abidjan, mais en raison de problèmes de distance qui se posaient au centre d'Adiopodoumé où, à dix-sept kilomètres d'Abidjan, étaient regroupées toutes les autres disciplines, il fut décidé d'installer les sciences humaines dans la concession qui avait servi de base à quelques enquêtes régionales lourdes en Côte-d'Ivoire, notamment celles de la SEDES [Société d'étude pour le développement économique et social], c'està-dire dans la zone 4 d'Abidjan qui deviendra, par la suite, le centre de PetitBassam des sciences humaines de l'ORSTOM. La même année, Emmanuel Terray fut nommé à l'université d'Abidjan, flambant neuve, mais je dois dire qu'il avait un peu d'appréhension à pénétrer, comme Marc Augé d'ailleurs, inside Africa... Ce qui ne l'empêcha pas de fonder quelques mois plus tard l'Institut d'ethnosociologie de l'université d'Abidjan et de travailler chez les Dida ${ }^{4}$, puis deux ans plus tard chez les Abron, dont il allait tiré sa thèse d'État ${ }^{5}$.

2. Voir Jean-Louis Boutillier, La Moyenne vallée du Sénégal (étude socio-économique), Paris, PUF, 1962.

3. Jean-Louis Boutillier, Bongouanou, Côte-d'Ivoire. Étude socio-économique d'une subdivision, Paris, Berger-Levrault, 1960 ("L'Homme d’outre-mer » nouv. sér. 2).

4. Cf. Emmanuel Terray, L'Organisation sociale des Dida de Côte-d'Ivoire, Abidjan, Annales de l'université d'Abidjan, 1969. Voir également les entretiens d'Emmanuel Terray avec Jean-Paul Colleyn, Traversées. Livres, action et voyages, Bruxelles, Éd. Labor, $2005: 83$ sq.

5. Cf. Emmanuel Terray, Une histoire du royaume abron du Gyaman, Paris, Karthala, 1995. 
En 1966, il n'y avait que quelques boîtes de nuit à Treichville [quartier populaire d'Abidjan], et on s'y retrouvait tous à danser le samedi soir. Il faut dire que l'on sortait des "années de plomb" en Côte-d'Ivoire, pendant lesquelles nombre d'écrivains et intellectuels ivoiriens furent arrêtés et emprisonnés par Houphouët-Boigny ${ }^{6}$. Je me souviens d'un méchoui chez Marc Augé (qui était arrivé au milieu de 1965) où il y avait Harris Memel-Fôte, Georges Niangoran-Bouah, Bernard Dadié, Amadou Hampâté-Bâ ; donc, il y avait là un mélange qui contredisait l'idée reçue que les Africains n'étaient pas invités à dîner chez les Blancs ou ne voulaient pas aller chez eux...

Je ne connaissais pas Marc Augé avant sa venue en Côte-d'Ivoire. Ce qui n'était pas le cas d'Emmanuel Terray qui, lui, l'avait côtoyé à l'École normale, mais on ne peut pas dire qu'à l'époque existait une particulière affinité entre eux, même sils avaient suivi ensemble les cours de Georges Balandier. En fait, Marc Augé était plus lié à Pierre Bonnafé, et c'est celui-ci qui l'avait emmené suivre les cours de Georges Balandier. Le choix des Alladian - le premier terrain de Marc Augé - s'est fait un peu au hasard. Il y avait, comme on dit, des trous à combler dans la connaissance ethnologique des populations ivoiriennes, et je me rappelle l'avoir laissé dans une case d'un village alladian, non sans un certain courage de sa part, après une première virée en voiture. Pierre Étienne était déjà là, qui, dans la foulée des enquêtes SEDES, travaillait sur la région baoulé, autour de Bouaké. Et il y avait aussi tout un groupe de gens qui fréquentaient Petit-Bassam, tels que la géographe Anne-Marie Cotten, l'historienne Claude-Hélène Perrot, le sociologue et anthropologue Jean-Marie Gibbal, etc. Linnovation fut que, une fois par mois, nous nous réunissions, écoutions un exposé fait par l'un d'entre nous ou par l'un de nos collègues ivoiriens, et discutions en buvant des verres sur les pelouses accueillantes du centre de Petit-Bassam. Plus tard, dans la soirée, nous allions dîner en groupe dans un "maquis" ou un restaurant vietnamien. Je me rappelle encore qu'une fois Emmanuel Terray y avait lu à haute voix des passages $d u$ Petit Livre Rouge, tels que "La contradiction au sein du peuple" ou "La révolution n'est pas un dîner de gala...", etc.

Dans ces années-là, il y eut une grande date : décembre 1967, le congrès des africanistes à Dakar, en présence d'Alioune Diop, le fondateur de Présence africaine, où nous avions affirmé que la recherche devait désormais se faire par des Africains. Puis est venu Mai 1968. Et le directeur général de l'OSTORM

6. Jean-Louis Boutillier fait ici allusion à ce que l'on a appelé les «pseudo-complots» contre le régime d'Houphouët-Boigny en 1963-1964 et qui permit à celui-ci de réprimer une bonne partie de l'élite intellectuelle et politique ivoirienne et de conforter son système de parti unique. 
avait compris qu'il fallait donner l'indépendance, y compris budgétaire, au centre de Petit-Bassam, dont je devins alors le premier directeur jusqu'en 1970. Mais la plupart du temps, les réunions du centre étaient informelles. Les discussions autour de l'anthropologie dite marxiste - dont on peut croire, rétrospectivement, qu'elle occupait au premier chef les cercles parisiens étaient en fait assez peu présentes, chacun menant sa recherche en toute indépendance: je n'avais pas une conception très autoritaire, ni matérielle ni intellectuelle, de la direction d'un centre de recherches. Ce n'est qu'en 1974, au moment de la parution du livre-manifeste Qui se nourrit de la famine en Afrique? (Paris, Maspéro) que mes rapports avec le directeur général de l'ORSTOM se sont détériorés, celui-ci m'ayant interdit de remettre les pieds en Afrique noire après avoir su que javais collaboré à cet ouvrage.

Rétrospectivement, je dirai que nous nous sommes comportés comme des petits-bourgeois installés dans un néocolonialisme de fait, mais avec une bonne conscience d'anticolonialistes. En vérité, c'était une sorte de bulle... On était confortés dans notre bonne conscience parce qu'on avait des rapports plutôt bons avec les Africains, aussi bien en ville que dans les villages, aussi bien à l'université qu’à la maison (on employait des boys que l'on payait bien...). Quand je pense à ce qu'on a pu faire dans la tranquillité de la brousse ou la relative quiétude des rues abidjanaises! Le pays était pacifique et sans danger. Quand jallais à Bobo-Dioulasso, en Haute-Volta (aujourd'hui Burkina Fasso), je traversais des zones pleines des hautes herbes d'après l'hivernage, où rien ne marrivait, jamais: les gens vous poussaient quand vous étiez embourbé ou ensablé, etc.

Au fond, cette période me laisse un goût bizarre dans la bouche, car tout sinscrivait dans un entre-deux, c'est-à-dire entre une période coloniale dure et une non moins dure période postcoloniale, dont on n'eut, à l'époque, ni la moindre perception, ni la moindre idée, ni la moindre prémonition, autrement dit celles des débuts de la mondialisation, de l'invasion des Églises évangéliques (qui était alors perçues comme du folklore), etc.

Mes souvenirs sont en réalité un peu équivoques. Avec le recul des années, cela me donne d'ailleurs le vertige. Tout cela a changé à partir des années 1980. En somme, on aurait pu parler de tas de problèmes, mais ça n'a pas été le cas. Nos maîtres ont été un peu irresponsables, assez inconscients de l'histoire en train de se faire, nous embarquant, par exemple, dans des enquêtes de terroir sans fin... Il y eut, incontestablement, un manque de lucidité de leur part tout autant que de la nôtre. Nous ne pensions pas les problèmes d'un point de 
vue profond. Des pans entiers de la recherche ont été laissés en friche: l'éducation, les classes sociales en Côte-d'Ivoire, le développement du capitalisme, la politique foncière, la commercialisation du café et du cacao, les problèmes de nationalité, etc.

Je suis parti de Petit-Bassam en 1970. Marc Augé était rentré en France (il devait faire plusieurs missions en Côte-d'Ivoire par la suite, mais pas de séjours longs comme ce fut le cas en 1965-1967). Emmanuel Terray était lui aussi rentré, mais en quelque sorte de force, son départ ayant été demandé par l'ambassadeur de France sous prétexte de "sentiments antifrançais" qu'il aurait exprimés.

Institut de recherche pour le développement (ex-ORSTOM), Paris. 